\title{
Multi-objective optimization of grooved gas journal bearings for robustness in manufacturing tolerances
}

\author{
Eliott Guenat, Jürg Schiffmann \\ Ecole Polytechnique Fédérale de Lausanne, Laboratory for applied \\ mechanical design, Maladière 71b, CP 526, CH-2002 Neuchâtel 2
}

\begin{abstract}
A tolerancing method highlighting trades-off against key design variables of mechanical systems is proposed and applied to herringbone-grooved gas journal bearings. Gas bearings typically suffer from a subsynchronous instability demanding a very tight tolerance on the bearing clearance and the groove depth. Classical optimization techniques look for the most stable design, which does not necessarily lead to most robust design against manufacturing deviations. The proposed method uses a non-dimensional multidimensional look-up table of stability score (critical mass), covering a large design space of gas bearings. It then dimensionalizes the table for a specific rotor-bearing system, highlighting regions of the hyperspace where the system is stable. The hyperspace is sliced into 2-D maps and a Monte-Carlo method creates windows within the stable domain along the two most critical design variables regarding manufacturing: the bear-
\end{abstract}


ing clearance and the groove depth. Width and length of the windows represent the manufacturing tolerance allowed on the two parameters to remain stable. A Pareto front of optimum windows in the entire hyperspace is then compiled. It displays the trade-off between the tolerance against deviation in clearance and groove depth, allowing the designer to select a nominal geometry tailored to the available manufacturing methods. A test rotor is analyzed with this method and the effects of pressure, speed, viscosity, radius, mass and centrifugal growth on manufacturing tolerances are investigated, highlighting that the radius and the viscosity have the greatest impact on the robustness.

\section{KEYWORDS}

Gas Bearing; Herringbone-groove journal bearing; Design; Manufacturing Tolerances

\section{INTRODUCTION}

Recent years have seen a strong development of high-speed turbomachines supported on gas bearings, for example for heat pumping $[1,2]$ or fuel cell air supplies or recirculation devices [3]. Among the gas bearing technologies accompanying this development, Herringbone Grooved Journal Bearings (HGJB) have 
the advantage of being reliably modelled and repeatable regarding manufacturing, characteristics still missing for compliant foil gas bearings [4]. However, the manufacturing tolerances necessary to achieve stable operation on rigid bearings represent a serious economic bottleneck limiting the large-scale marketability of HGJB-supported machines. Although several authors have investigated the sensitivity of stability in gas bearings to manufacturing errors $[5,6,7]$, the literature lacks systematic design strategies to maximize the stability robustness.

\section{Nature of the issue}

The optimization and integrated design of turbomachinery supported on HGJB is typically adopting a mono or multi-objective strategy comprising the maximization of the gas bearing stability, either in terms of critical mass [8], or logarithmic decrement [2]. Following the development of the Narrow-Groove Theory [9], HGJBs were first optimized to maximize the load capacity $[9,10]$. Later, the optimization of HGJB was oriented toward a maximization of the stability based on the critical mass criterion $[11,8]$. More recent years have seen the emergence of multi-objective, integrated optimal design of entire rotor-HGJB systems [12], also including the spiral groove thrust bearing in the optimization. Typical competing objectives are the minimization of losses and the maximization of a sta- 
bility criterion. The underlying assumption is that a high nominal stability of the HGJB supported rotor will also allow a large deviation of the bearing parameters from their design point, while keeping the system dynamically stable. However, achieving an accurate bearing clearance through lapping or grinding is a challenging and expensive task. The manufacturing of the grooves through laser or chemical etching, grinding or even additive manufacturing [13], generally yields accurate groove width, length and angle. The groove depth, however, usually exhibits a large relative uncertainty $[14,15]$. Since manufacturing deviation of the bearing clearance and groove depth can easily achieve $\pm 15 \%$, the effect of this wide dimensional range can have a significant impact on stability and load capacity. As a consequence, rather than simply maximizing the stability, the maximization of tolerances, might lead to a more manufacturing-oriented design procedure for HGJB, thus improving their cost effectiveness. Meanwhile, design tools and concepts such as multi-objective optimizations and Pareto fronts have already proven their suitability in the tolerancing of mechanical systems $[16,17]$ and might be of interest for the tolerancing of aerodynamic bearings. 


\section{Goals and objectives}

The present work aims to 1) propose a multi-objective optimization procedure for maximizing the range of selected design parameters while minimizing their effect on selected performance metrics, 2) apply the methodology to the design of a gas bearing supported rotor and 3) use the results to devise design guidelines for the maximization of the manufacturing tolerances for HGJB.

\section{Scope of the Paper}

The proposed optimization procedure for improving the design robustness with regards to deviation is based on the establishment of a non-dimensional performance matrix that is computed only once and that serves as a look-up table for all similar design problems. For each particular design case, the non-dimensional performance matrix is transformed into the dimensional space according to the specified design problem. Hence, this methodology can be used for any design problem that can be expressed with non-dimensional numbers such as turbomachinery, gas lubricated bearings or heat exchangers among many. In this paper, the authors suggest to look at the design of herringbone grooved journal bearings. The HGJB model based on the Narrow Groove Theory under the assumption of concentric position is used to build a dimensionless stability look- 
up table covering a large domain of bearing geometries. For a particular design case, this table is then dimensionalized for a specific rotor-bearing system and the minimum critical mass criterion of Fleming and Harmock [8] is employed to determine the whirl stability. The resulting matrix is sliced along each of its dimensions and a Monte-Carlo method generates a Pareto front of manufacturing tolerances in clearance and groove depth at each slice. Finally, the several slice fronts are combined in a global Pareto front representing the maximum achievable manufacturing tolerances. The method is applied on a case rotor and the sensitivity of the final Pareto front on rotor and ambient parameters is analyzed. Based on the results, general design guidelines are devised.

\section{THEORY}

The strategy to perform an optimization maximizing the manufacturing tolerances on design variables of HGJB consist in computing a stability criteria within a large non-dimensional design space, where each dimension of this space is a key variable of the gas bearing. Typically, the geometry of a classical HGJB can be described by 6 non-dimensional variables: the groove aspect ratio $\alpha$, the

groove angle $\beta$, the relative land length $\gamma$, the relative groove depth $\bar{\delta}$, the bearing length-diameter ratio $L / D$ and the compressiblity number $\Lambda$. The 6-D space 
is discretized and and the non-dimensional stability is computed for each node using the critical mass criteria. This multi-dimensional matrix is the base that serves as a look-up table to investigate the performance of any given rotor supported on HGJB. To study a particular rotor-bearing system undergoing an acceleration from rest to full speed, the stability is evaluated at discrete speeds in this range and the minimum stability achieved along this path is retained. This step requires the dimensionalization of the hyperspace previously computed, using characteristics of the rotor (radius, mass, clearance, groove depth), the ambient conditions (pressure, viscosity) and the operating conditions (angular velocity). This transformation into the dimensional space allows to introduce clearance distortion effects (thermal and centrifugal) and the absolute manufacturing deviations. For each of the $n$ speed steps along the acceleration path, a dimensional hyperspace is created from the generic non-dimensional one (Figure .1). The $n$ hyperspaces are combined to retain the minimum critical mass at each node, leading to one single rotor-specific hyperspace used to express the minimum critical mass. Two of its design dimensions are kept dimensional: the bearing ridge clearance $h_{0}$ and the groove depth $\delta$, since these are the critical variables for the manufacturing process. The 4 other dimensions are still non-dimensional: $\alpha, \beta, \gamma$ and $L / D$. 
The next step consists in the slicing the specific dimensional hyperspace to extract planes along the bearing clearance and the groove depth. On each slice (constant $\alpha, \beta, \gamma$ and $L / D$ ), a Monte Carlo method is used to generate a large number of rectangles aligned on the axis of nominal clearance and groove depth, whose geometrical center is in the stable domain. Each rectangle is defined by 4 parameters: the position of its center along the clearance and groove depth $\left(h_{0, \square}\right.$ and $\delta_{\square}$ respectively) and its half length along each axis ( $\Delta h_{0}$ and $\left.\Delta \delta\right)$. Each rectangle undergoes a verification to ensure it satisfies the performance criterion $\left(m_{\text {rotor }}<m_{c, \min }\right)$, the outliers being discarded. The validation algorithm checks 12 points on a grid centred on the rectangle and 8 points randomly scattered in the rectangle (Figure .2). Valid rectangles are represented in a 2D space defined by their length along the two directions, which represents the viable sets of manufacturing tolerances to obtain a stable operation of the specific rotor-bearing system:

$$
\begin{gathered}
h_{0}=h_{0, \square} \pm \Delta h_{0} \\
\delta=\delta_{\square} \pm \Delta \delta
\end{gathered}
$$

The collection of all these tolerance windows allows to identify a Pareto front of optimum solutions in terms of maximal dimensional deviation and its constitutive points are stored. This procedure, depicted in Figure .3, is repeated for each 
slice of the discrete hypervolume. Finally, all the particular fronts are combined to obtain the global Pareto front for the particular system, as illustrated in Figure .4 .

The global front represents the feasible manufacturing tolerances regarding the nominal clearance and the groove depth for a particular rotor, while ensuring stable operation (i.e. satisfaction of a minimal performance metric). Depending on the accuracy of the considered manufacturing techniques, designers can then select a solution along the Pareto front suiting best their needs and possibilities, depending on the actual context.

\section{Bearing model}

The HGJB can be efficiently modelled by the Narrow Groove Theory introduced by Vohr and Pan, which assumes an infinite number of grooves along the bearing cricumferential direction. Lund's perturbation method [11] can be applied around the equilibrium position to predict the frequency-dependent impedances and then determine the critical mass for the whirl stability. The NGT is applied here assuming the following points:

- The bearing is operated at a concentric positions

- The lubricant is an ideal gas in laminar regime 
- The fluid film is isothermal and isoviscous

- Ambient conditions are met at the axial bearing boundaries $(\bar{z}= \pm L / D)$

- No axial mass flow at $\bar{z}=0$ (bearing symmetry)

The modified Reynolds equation obtained from the NGT is fully developed in [12]. The final non-dimensional expression is repeated in Eq. 3 in cylindrical coordinates, with the coefficients explicited in the Appendix.

$$
\begin{array}{r}
\partial_{\theta}\left[\bar{P}\left(f_{1} \partial_{\theta} \bar{P}+f_{2} \partial_{\bar{z}} \bar{P}\right)\right]+\partial_{\bar{z}}\left[\bar{P}\left(f_{2} \partial_{\theta} \bar{P}+f_{3} \partial_{\bar{z}} \bar{P}\right)\right] \\
+c_{s}\left(\sin \psi \partial_{\theta}\left(f_{4} \bar{P}\right)-\cos \psi \partial_{\bar{z}}\left(f_{4} \bar{P}\right)\right) \\
-\Lambda \partial_{\theta}\left(f_{5} \bar{P}\right)-\sigma \partial_{\bar{t}}\left(f_{5} \bar{P}\right)=0
\end{array}
$$

The general nomenclature of the HGJB is summarized in Figure .5.

Lund's method is applied and the critical mass is obtained, following the procedure described in [18]. The critical mass defines the theoretical mass a bearing can support at a given compressibility number before being unstable. It is nondimensionalized as follows:

$$
\bar{m}_{c r i t}=\frac{m_{c r i t} P_{a} h_{0}^{5}}{2 L R^{5} \mu^{2}}
$$

The critical mass of HGJB is not necessarily decreasing with the compressibility number. Therefore, the minimum value of the critical mass between rest and maximum compressibility number has to be evaluated. 
The prediction of the minimum critical mass is tested with the numerical data of Fleming and Hamrock [8] who optimized the geometry of HGJB to maximize this metric. The results, showed in Figure .6 for a bearing with $L / D=1$ and the grooved member rotating, exhibit a maximum relative deviation of $1.4 \%$.

\section{Experimental validation}

The concept of NGT-predicted critical mass has been used by the authors for the design of numerous prototypes of high speed HGJB-supported rotors operating at different compressibility numbers. The parameters of the bearings are reported in Table .1, with the ratio between predicted minimum critical mass and actual rotor mass $\left(M_{r}=\bar{m}_{c, \min } / \bar{m}_{\text {rotor }}\right)$ reported in Figure .7 against the maximum compressibility number. All the reported systems achieved stable operation up to nominal speed, which supports the reliability of the model to practically predict the stability of HGJB-rotor systems.

\section{RESULTS AND DISCUSSION}

\section{Computation of the non-dimensional design space}

The computation of the universal non-dimensional 6D stability matrix is computationally expensive, since it can easily exceed $10^{10}$ evaluation points to obtain 
a sufficiently tight grid. In order to reduce this amount, the present analysis excludes the parameters $\gamma$ and $L / D$, which are both set to 1 to maximize the stability of a bearing with the grooved member rotating [8]. The matrix is constituted of $3.2 \cdot 10^{7}$ elements in the following ranges of parameters:

$$
\begin{array}{lr}
\Lambda \in[0,200] & 450 \text { steps } \\
\alpha \in[0.4,0.6] & 10 \text { steps } \\
\beta \in\left[10^{\circ}, 80^{\circ}\right] & 70 \text { steps } \\
\bar{\delta} \in[0,8] & 100 \text { steps }
\end{array}
$$

\section{Case study of the gas bearing supported rotor}

A case rotor is defined for the dimensionalization of the pre-computed 4-D stability matrix and the determination of the optimal manufacturing tolerances. The rotor is taken as symmetric in order to have half the mass supported by each of the two journal bearings. Its main characteristics are listed in Table .2. In a first approach, the centrifugal growth of the rotor is neglected. The critical mass is evaluated for 10 speeds between standstill and the maximum regime, in 
the following range of parameters:

$$
\begin{aligned}
& h_{0} \in[4 \mu m, 13 \mu m] \\
& \delta \in[2 \mu m, 28 \mu m] \\
& \alpha \in[0.4,0.6] \\
& \beta \in\left[10^{\circ}, 80^{\circ}\right]
\end{aligned}
$$

The lower bound of the clearance was chosen as a limit beyond which rarefied gas effects should have been considered. A groove depth of $0 \mu \mathrm{m}$ was not investigated, since it corresponds to a plain bearing, which is unstable at concentric position. In each slice of the final stability matrix, $3 \cdot 10^{5}$ random tolerance windows are generated, whose centers are located in the stable domain of the slice, if any. An example of the stability limit for three values of groove angle is shown in the groove depth-clearance domain in Figure .8 , for $\alpha=0.6$. The arrows along the curves indicate the orientation towards the stable domain. It is interesting to note that for angles below 25 the stable region is fully contained between the corresponding contour. However, for an angle of 28 the topology of the feasible region changes. The stable region is a more complex one since it is delimited by two concentric contours, yielding an unstable region centered around the considered range of clearance and groove depth. The advantage of 
the implemented methodology is that it can easily handle such changes in topology of the mathematical response surface.

\section{Nominal case}

For the nominal rotor, the tolerancing algorithm outputs the Pareto curve shown in Figure .9. The Pareto curve indicates clearly that the two tolerances are competing: they can not be maximized both simultaneously. The maximum tolerance on the groove depth is roughly 5 times larger than the tolerance on the bearing clearance, indicating a lower sensitivity of the stability on this parameter. The extremities of the front are not particularly interesting from a manufacturing point of view, since a small concession on the maximized variable allows a large gain on the other. In this particular case, if one targets a large clearance tolerance, a reduction of $0.25 \mu \mathrm{m}$ on $\Delta h_{0}$ from its maximum value, allows to increase the tolerance of delta-groove depth from nearly 0 to $4.5 \mu \mathrm{m}$ on $\Delta \delta$.

The parameters $\alpha$ and $\beta$ of the points in the Pareto front are shown in Figure .10 . A rising trend for $\beta$ is visible as the tolerance on the clearance increases, ranging from $22^{\circ}$ to $27^{\circ}$. No specific trend is visible for $\alpha$, as optimal solutions are found over the entire range of this parameter. This suggests that although important when considering the maximum stability point, $\alpha$ is not as significant when 
considering the stability tolerance. The nominal clearance and groove depth values corresponding to the Pareto front are shown in Figure .11. The trend is rising on both values as the tolerance on the clearance increases. Past a value for $\Delta h_{0}$ of $0.5 \mu \mathrm{m}$, the trend for $h_{0, \square}$ follow a linear trend roughly expressed as follows: $h_{0, \square}=\Delta h_{0}+4 \mu \mathrm{m}$, meaning that the optimal tolerancing window is bounded by the lower limit for the bearing clearance set for this particular study. Interestingly, for $\Delta h_{0}<0.5 \mu \mathrm{m}$, this limit is not bounding the rectangles in the $h_{0}$ direction.

\section{Comparison with stability maximization}

For comparison purpose, the results of the optimization of the bearing parameters for the maximization of the stability without considering the effect of tolerancing is performed. In this case, the bearing nominal clearance is varied from 4 to $10 \mu \mathrm{m}$ and the set of $\alpha, \beta$ and $\delta$ maximizing the minimum critical mass along the speed range is found in the dimensional look-up table. The results are shown in Figure .12. The parameters clearly deviate from the one shown in Figures .10 and .11. At equal bearing clearance, the groove angle tend to be higher and the grooves deeper when maximizing the tolerance windows and not the stability. This highlights the fact that a pure optimization for the maximization of the crit- 
ical mass does not yield the same bearing parameters as an optimization maximizing the tolerances along $h_{0}$ and $\delta$ under the constraint of stable operation, therefore justifying a dedicated optimization procedure.

\section{Sensitivity analysis}

Starting from the studied rotor, the influence of the dimensionalization parameters on the manufacturing tolerances are studied. These parameters are the ambient pressure and viscosity, the rotor radius, mass and speed. Each parameter is varied of $\pm 20 \%$ and the resulting global Pareto fronts are computed. Results are visible in Figure .13 to .17 . The variations on the maximum achievable tolerances on the bearing clearance and groove depth are summarized in Figures .18 and .19 respectively. A variation on the radius has the largest effect on the tolerance windows with an relative variation on the maximum tolerances more than twice the radius relative variation. For the other parameters have an influence smaller than their own variation. For the specific rotor studied here, the maximum rotor speed exhibits a negligible effect on the tolerances, meaning that the stability bottleneck is not located at the maximum speed, but at a lower rotor speed. Considering that the presented strategy is based on the minimum critical speed encountered from rest to maximum speed, increasing the target speed can 
either have a negative effect or no effect at all, but cannot improve the manufacturing tolerances.

In relative proportions, the tolerance on the bearing clearance is systematically more sensitive to parametric variations than the groove depth tolerance, suggesting that the designers should wisely select the rotor parameters if the goal is to ensure a possibly large tolerance on the bearing clearance. As design guidelines, the results indicate that lighter rotors with a larger diameter at bearing locations, operating at lower ambient pressures and higher temperatures (associated with higher gas viscosities) allow to achieve a broader manufacturing tolerance.

\section{Effect of centrifugal growth}

Typical high-speed rotors undergo a centrifugal growth leading to a significant reduction of the bearing clearance. The centrifugal growth is proportional to $\Omega^{2}$, $R^{3}$ and to $\rho / E$. The effect of the centrifugal growth is investigated here by setting the absolute radial growth of the rotor at maximum speed and to scale it for the different speeds from rest to the target rotor speed as follows [1]:

$$
\Delta h_{\text {growth }}=\Delta h_{\text {growth }, \max } \Omega^{2} / \Omega_{\max }^{2}
$$

which is used to compute the actual clearance:

$$
h_{r}=h_{0}-\Delta h_{\text {growth }}
$$


The maximum expansion is set from 0 to $3 \mu \mathrm{m}$ with a $1 \mu \mathrm{m}$ interval. Results in form of Pareto fronts are represented in Figure .20. Centrifugal growth has a slight positive effect of the maximum clearance tolerance. At a radial expansion of $3 \mu \mathrm{m}$, the maximum tolerance field is $10.0 \%$ higher than the nominal case without centrifugal growth. Interestingly, the maximum groove depth tolerance remains unaffected. The centrifugal growth appears to have a stabilizing effect by reducing the effective clearance. However, as the stability bottleneck happens at a lower speed than the target speed and considering the quadratic dependency of the expansion with the angular velocity, the overall effect is modest.

\section{CONCLUSION}

A generalized method to perform a multi-objective optimization of a mechanical system for maximizing the manufacturing tolerances was presented. A computationallyintensive dimensionless look-up table of a feasibility criteria, covering all the design space is computed only once. This matrix is then converted into a dimensional, system-specific matrix at a very low computational cost. From this matrix, the Pareto front of competing manufacturing tolerances is found, allowing the designers to select the appropriate trade-off depending on the available resources. This data-driven methodology to identify the robustness limits with re- 
gards to dimensional deviation can be applied to any design problem that can be represented in a non-dimensional form. In this paper the method was applied to the problem of rotors supported on herringbone grooved journal bearings, where the critical mass is the feasibility criteria, and the clearance and groove depth the design parameter whose tolerance has to be maximized. A case rotor is investigated, and the influence of multiple rotor parameters on the manufacturing tolerances was analyzed. The conclusive remarks for this particular design problem are:

- The determination of the manufacturing tolerances on the clearance is competing with the tolerance on the groove depth

- The groove depth tolerance field is significantly larger than the tolerance range on the bearing clearance

- The classical maximization of critical mass does not yield the bearing parameters corresponding to an optimal manufacturing tolerances.

- Low ambient pressures, high gas viscosities, large rotor diameters and low rotor masses allow a larger manufacturing tolerance of the HGJB in both groove depth and bearing clearance

- The centrifugal growth of the rotor has a slight positive influence on the 
manufacturing tolerance of the bearing clearance but not on the groove depth

The presented method allows to progress toward a cost-oriented design of highspeed gas bearing supported rotors, improving the marketability of such machines.

\section{ACKNOWLEDGMENT}

The authors acknowledge the co-funding by the Swiss National Science Foundation, grant PYAPP2_154278/1. The authors have no conflict of interest to declare. 


\section{Reference}

[1] J. Schiffmann, D. Favrat, Integrated design and optimization of gas bearing supported rotors, Journal of Mechanical Design 132 (5) (2010) 051007. doi:10.1115/1.4001381.

[2] J. Schiffmann, Integrated design and multi-objective optimization of a single stage heat-pump turbocompressor, Journal of Turbomachinery 137 (7) (2015) 071002. doi:10.1115/1.4029123.

[3] P. H. Wagner, Z. Wuillemin, S. Diethelm, J. Van herle, J. Schiffmann, Modeling and designing of a radial anode off-gas recirculation fan for solid oxide fuel cell systems, Journal of Electrochemical Energy Conversion and Storage 14 (1) (2017) 011005. doi:10.1115/1.4036401.

[4] K. Shalash, J. Schiffmann, On the manufacturing of compliant foil bearings, Journal of Manufacturing Processes 25 (2017) 357-368. doi:10.1016/j.jmapro.2016.12.021.

[5] M. Song, S. Azam, J. Jang, S.-S. Park, Effect of shape errors on the stability of externally pressurized air journal bearings using semi-implicit scheme, Tribology International 115 (2017) 580-590. doi:10.1016/j.triboint.2017.06.037. 
[6] H. Cui, Y. Wang, X. Yue, M. Huang, W. Wang, Effects of manufacturing errors on the static characteristics of aerostatic journal bearings with porous restrictor, Tribology International 115 (2017) 246-260. doi:10.1016/j.triboint.2017.05.008.

[7] Y.-B. P. Kwan, J. B. Post, A tolerancing procedure for inherently compensated, rectangular aerostatic thrust bearings, Tribology International 33 (8) (2000) 581-585. doi:10.1016/S0301-679X(00)00109-2.

[8] D. P. Fleming, B. J. Hamrock, Optimization of self-acting herringbone journal bearing for maximum stability, 6th Gas bearing symposium.

[9] J. H. Vohr, C. Y. Chow, Characteristics of herringbone-grooved, gaslubricated journal bearings, Journal of Basic Engineering 87 (3) (1965) 568-576. doi:10.1115/1.3650607.

[10] V. Castelli, J. H. Vohr, Performance characteristics of herringbone- grooved journal bearings operating at high eccentricity ratios and with misalignment, Gas bearing symposium.

[11] J. W. Lund, Calculation of stiffness and damping properties of gas bearings, Journal of Lubrication Technology 90 (4) (1968) 793-803. doi:10.1115/1.3601723. 
[12] J. Schiffmann, Integrated design, optimization and experimental investigation of a direct driven turbocompressor for domestic heat pumps (2008). doi:10.5075/epfl-thesis-4126.

[13] E. Rasmussen, P. Rudolph, A. Mamishev, An experimental study of 3d printed herringbone grooved journal bearings, 2017. doi:10.1115/IMECE2017-73502.

[14] I. Ognjanovic, Experimental contribution to the mechanics of herringbone grooved journal air bearings (2011). doi:10.5075/epfl-thesis-4879.

[15] M. Ochiai, N. Kato, H. Hashimoto, Groove shape optimization of thrust air bearing for small size spindle considering the processing errors, in: Proceedings of the ASME-JSME 2018, 2018, p. 3.

[16] N. Jawahar, R. Sivasankaran, M. Ramesh, Optimal pareto front for manufacturing tolerance allocation model, Vol. 231, 2017, pp. 1190-1203. doi:10.1177/0954405415586548.

[17] J. Natarajan, R. Sivasankaran, G. Kanagaraj, Bi-objective optimization for tolerance allocation in an interchangeable assembly under diverse manufacturing environment, The International Journal of Advanced Manufacturing Technology 95 (5) (2018) 1571-1595. doi:10.1007/s00170-017-1232-y. 
[18] E. Guenat, J. Schiffmann, Real-gas effects on aerodynamic bearings, Tribology International 120 (2018) 358-368. doi:10.1016/j.triboint.2018.01.008. 


\section{Nomenclature}

\section{Roman symbols}

$a$

$b$

$c_{S}$

D

$f$

$g$

$h$

$h_{0}$

$h_{g}$

$h_{r}$

$L$

$M, m$

$N$

$P$

$R$

$z$
Groove length

Ridge length

NGT coefficient

Diameter

NGT coefficient

NGT coefficient

Clearance

Nominal clearance

Groove clearance

Ridge clearance

Bearing axial length

Rotor mass

Rotor speed

Pressure

Radius

Axial coordinate

\section{Greek symbols}




\begin{tabular}{|c|c|}
\hline$\alpha$ & Groove aspect ratio $a /(a+b)$ \\
\hline$\beta$ & Groove angle \\
\hline$\gamma$ & Land over length ratio $\left(1-L_{\text {land }} / L\right)$ \\
\hline$\Delta h_{0}$ & Tolerance on the nominal clearance \\
\hline$\Delta \delta$ & Tolerance on the groove depth \\
\hline$\delta$ & Groove depth \\
\hline $\bar{\delta}$ & Relative groove depth $\delta / h_{0}$ \\
\hline$\theta$ & Circumferential coordinate \\
\hline$\Lambda$ & Compressibility number \\
\hline$\mu$ & Dynamic viscosity \\
\hline$\sigma$ & Squeeze number \\
\hline$\Omega$ & Angular velocity \\
\hline & Superscripts \\
\hline- & Non-dimensional \\
\hline & Subscripts \\
\hline$a$ & Ambient condition \\
\hline crit & Critical \\
\hline$g$ & Groove \\
\hline growth & Cetrifugal growth \\
\hline
\end{tabular}




$\begin{array}{ll}\min & \text { Minimum } \\ \text { nom } & \text { Nominal } \\ r & \text { Ridge, ratio } \\ \text { rotor } & \text { Rotor } \\ \square & \text { Nominal } \\ & \text { Acronyms } \\ & \text { HGJB }\end{array}$




\section{Appendix}

The terms composing equation 3 are developed here. 


$$
\begin{aligned}
& \bar{h}_{r}=\frac{h_{r}}{h_{0}} \\
& \bar{h}_{g}=\frac{h_{g}}{h_{0}} \\
& g_{1}=\bar{h}_{g}^{3} \bar{h}_{r}^{3} \\
& g_{2}=\left(\bar{h}_{g}^{3}-\bar{h}_{r}^{3}\right)^{2} \alpha(1-\alpha) \\
& g_{3}=(1-\alpha) \bar{h}_{g}^{3}+\alpha \bar{h}_{r}^{3} \\
& c_{s}=-\frac{6 \mu \Omega R^{2}}{p_{a} h_{0}^{2}} \alpha(1-\alpha) \bar{\delta} \sin \psi \\
& f_{1}=\frac{g_{1}+g_{2} \sin ^{2} \psi}{g_{3}} \\
& f_{2}=\frac{g_{2} \sin \hat{\beta} \cos \psi}{g_{3}} \\
& f_{3}=\frac{g_{1}+g_{2} \cos ^{2} \psi}{g_{3}} \\
& f_{4}=\frac{\bar{h}_{g}^{3}-\bar{h}_{r}^{3}}{g_{3}} \\
& f_{5}=\alpha \bar{h}_{g}+(1-\alpha) \bar{h}_{r} \\
& \Lambda=\frac{6 \mu \Omega R^{2}}{P_{a} h_{r}^{2}} \\
& \sigma=\Lambda \frac{\omega}{\Omega}
\end{aligned}
$$




\section{Tables}

Table .1: Non-dimensional parameters of stable HGJB-supported prototypes tested by the authors

\begin{tabular}{lllllllll} 
Case & $\alpha$ & $\beta\left[^{\circ}\right]$ & $\gamma$ & $\bar{h}_{g}$ & $L / D$ & $\Lambda$ & $\bar{m}_{\text {crit,min }}$ & $\bar{m}_{\text {rotor }}$ \\
\hline 1 & 0.6 & 15 & 1 & 3.00 & 1 & 46.00 & 0.57 & 0.21 \\
2 & 0.6 & 15 & 1 & 3.00 & 1 & 27.10 & 4.41 & 0.57 \\
3 & 0.55 & 41 & 0.95 & 2.60 & 1.8 & 20.67 & 2.99 & 0.64 \\
4 & 0.6 & 20 & 1 & 2.75 & 1 & 38.04 & 10.77 & 0.65 \\
5 & 0.65 & 19 & 1 & 2.71 & 1 & 17.85 & 7.31 & 2.45 \\
6 & 0.65 & 20 & 1 & 2.60 & 1 & 10.44 & 4.17 & 1.97 \\
7 & 0.74 & 10 & 0.75 & 3.13 & 1.175 & 28.48 & 1.47 & 0.93 \\
8 & 0.65 & 22 & 1 & 3.14 & 1 & 7.825 & 15.53 & 6.31 \\
9 & 0.65 & 19.1 & 1 & 3.40 & 1 & 12.11 & 12.42 & 1.37 \\
11 & 0.65 & 17 & 1 & 3.89 & 1 & 20.77 & 8.76 & 0.91 \\
10 & 0.65 & 17 & 1 & 3.89 & 1 & 19.63 & 3.42 & 0.71
\end{tabular}


Table .2: Parameters of the studied rotor

$$
\begin{array}{rl}
R & 5 \mathrm{~mm} \\
m_{\text {rotor }} & 0.075 \mathrm{~kg} \\
N_{\max } & 200 \mathrm{krpm} \\
P_{a} & 10^{5} \mathrm{~Pa} \\
\mu_{a} & 18.5 \mu \mathrm{Pa} \cdot \mathrm{s}
\end{array}
$$




\section{List of Figures}

.1 Case-specific dimensionalization of the universal stability lookup table . . . . . . . . . . . . . . . . 33

.2 Location of the rectangle stability check points to verify that the rectangle covers no unstable points. Check points are either on a grid (black points) or randomly positioned (grey points). . . . . . 34

.3 Simplified representation of a stable domain on a slice (a), random generation rectangles in the stable domain (b) and determination of the particular Pareto front (c) . . . . . . . . . 35

.4 Particular Pareto fronts projected to obtain the global front . . . . 36

.5 Description and nomenclature of the HGJB geometry . . . . . . 37

.6 Comparison of the present bearing model with the literature on the computation of the minimum critical mass . . . . . . . . 38

.7 Predicted critical mass ratio against compressibility number for 12 HGJB-supported prototypes designed by the authors . . . . . . 39

.8 Examples of stability limits for the considered rotor and $\alpha=0.6$. The arrows indicate the orientation of the stable domain . . . . . . 40

.9 Global Pareto front for the nominal rotor-bearing system _ . . . . 41

.10 Values of $\alpha$ and $\beta$ resulting in the points shown in the Pareto front of Figure .9. . . . . . . . . . . . . . . 42

.11 Values of $h_{0, \square}$ and $\delta_{\square}$ resulting in the points shown in the Pareto front of Figure .9. . . . . . . . . . . . . . . . . 43

.12 Output parameters from the optimization maximizing the critical mass for different values of $h_{0} \ldots \ldots \ldots \ldots$. . . . . . . 44

.13 Effect of the ambient pressure on the global Pareto front . . . . 45

.14 Effect of the gas viscosity on the global Pareto front . . . . . . . 46

.15 Effect of the rotor radius on the global Pareto front . . . . . . . 47

.16 Effect of the rotor mass on the global Pareto front . . . . . . . 48

.17 Effect of the rotor maximum speed on the global Pareto front . . . 49

.18 Effect of parametric variation on the maximum clearance tolerance 50

.19 Effect of parametric variation on the maximum groove depth tolerance . . . . . . . . . . . . . . . . 51

.20 Effect of the centrifugal rotor radial growth on the tolerance Pareto Front . . . . . . . . . . . . . . . . . 52 


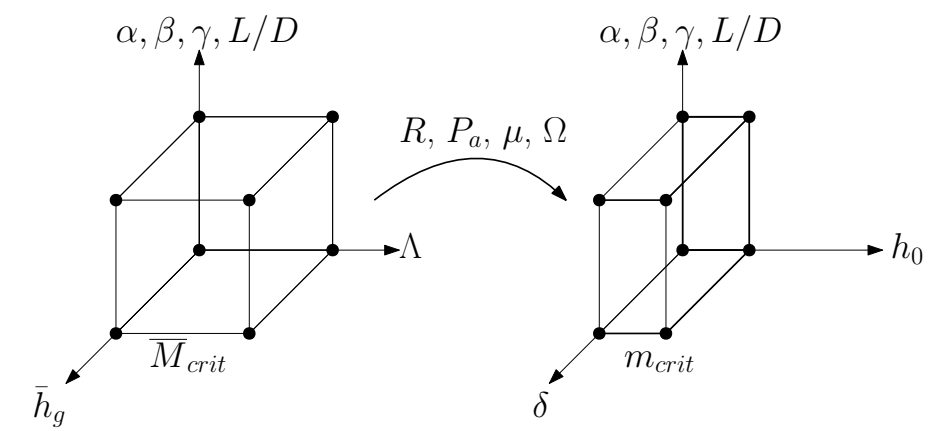

(a) Dimensionalization

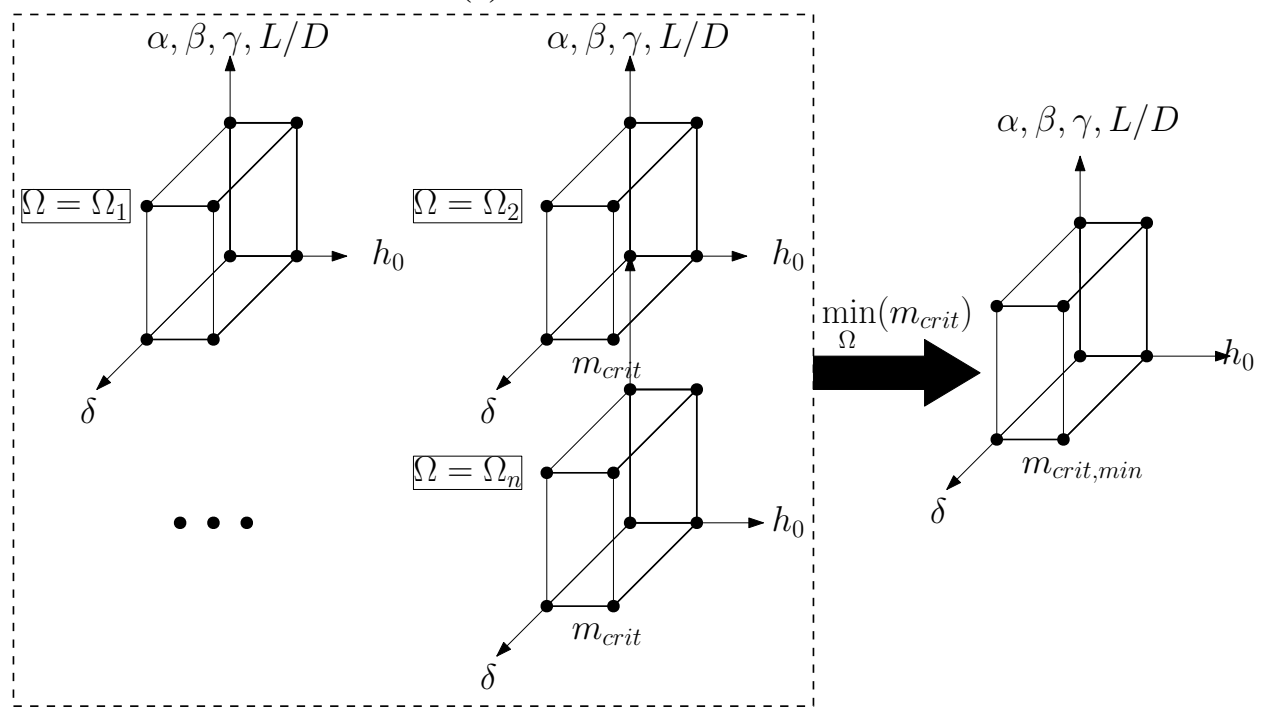

(b) Point-wise selection of the minimum critical mass

Figure .1: Case-specific dimensionalization of the universal stability look-up table 


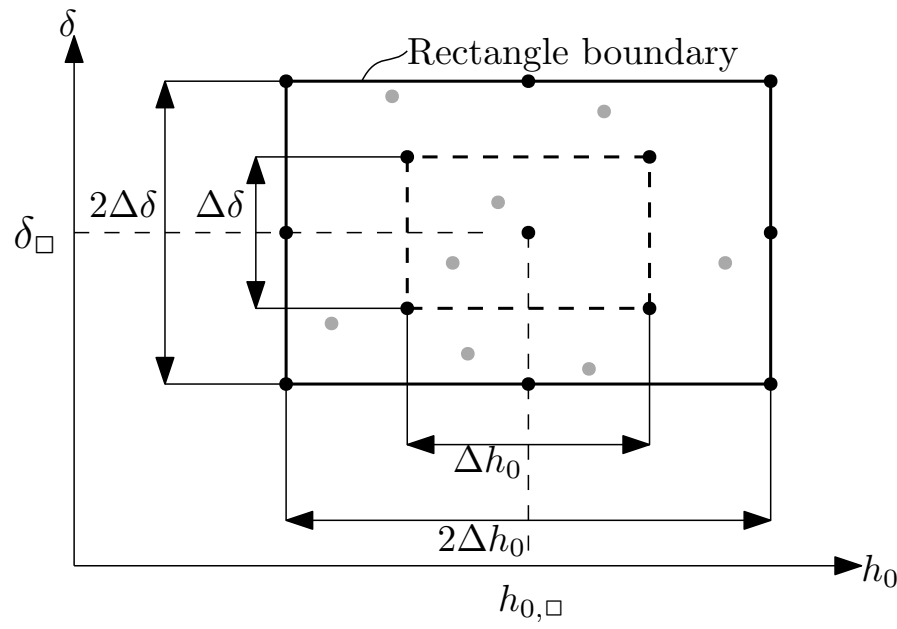

Figure .2: Location of the rectangle stability check points to verify that the rectangle covers no unstable points. Check points are either on a grid (black points) or randomly positioned (grey points). 


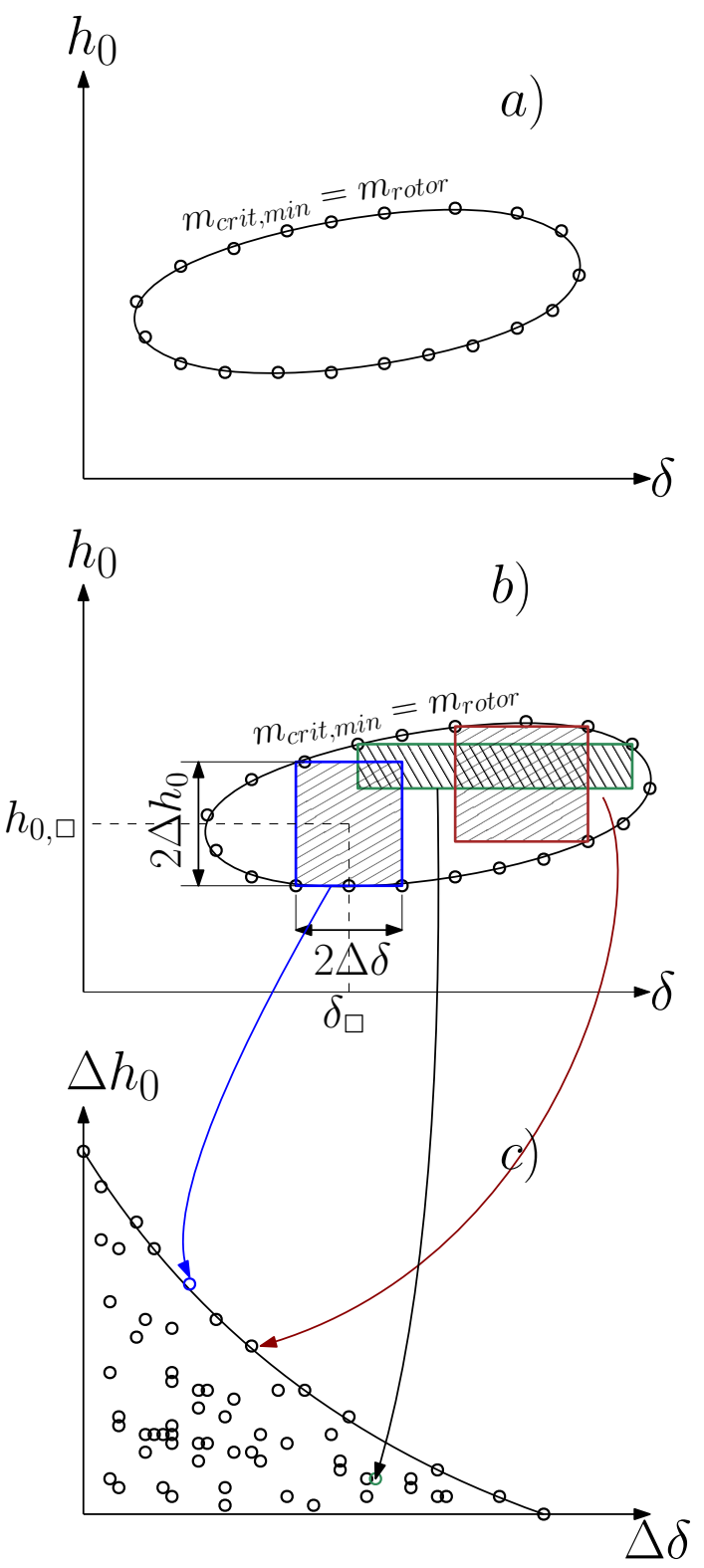

Figure .3: Simplified representation of a stable domain on a slice (a), random generation rectangles in the stable domain (b) and determination of the particular Pareto front (c) 


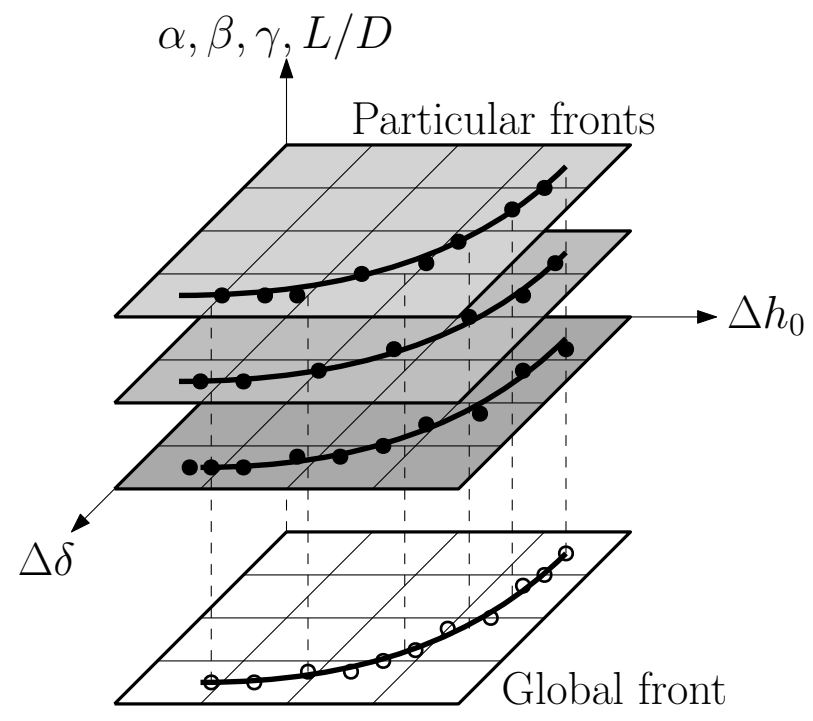

Figure .4: Particular Pareto fronts projected to obtain the global front 


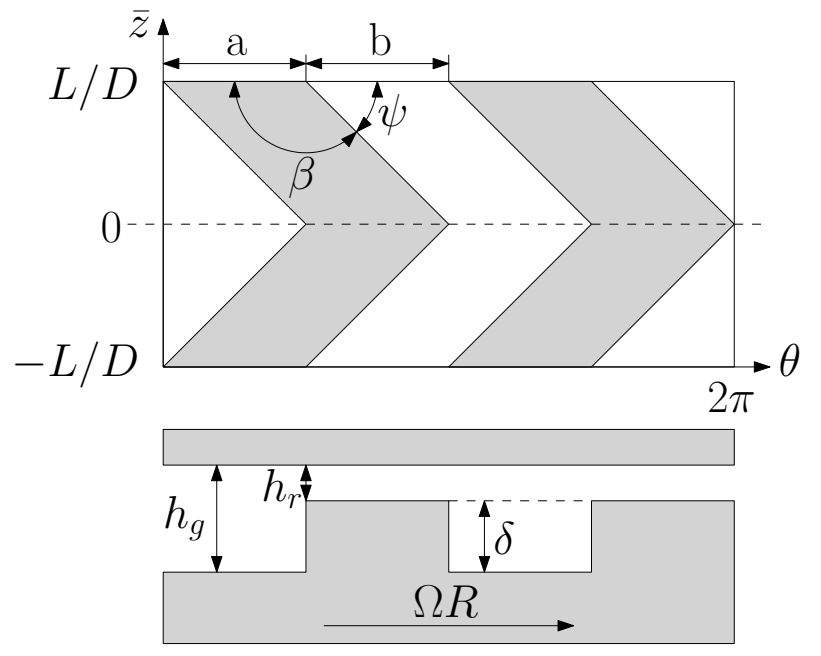

Figure .5: Description and nomenclature of the HGJB geometry 

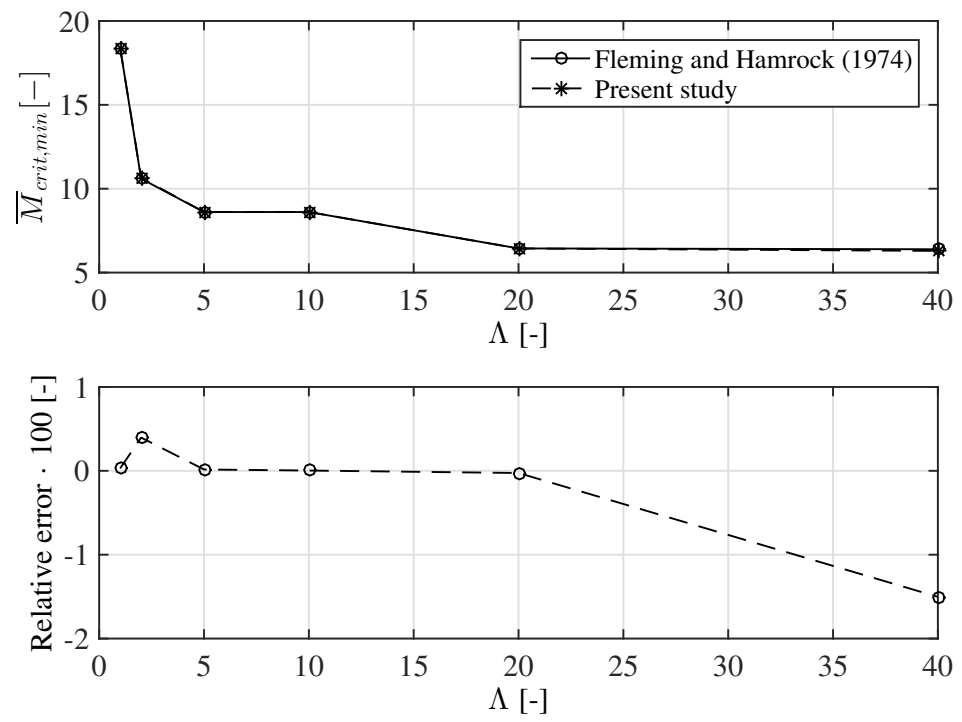

Figure .6: Comparison of the present bearing model with the literature on the computation of the minimum critical mass 


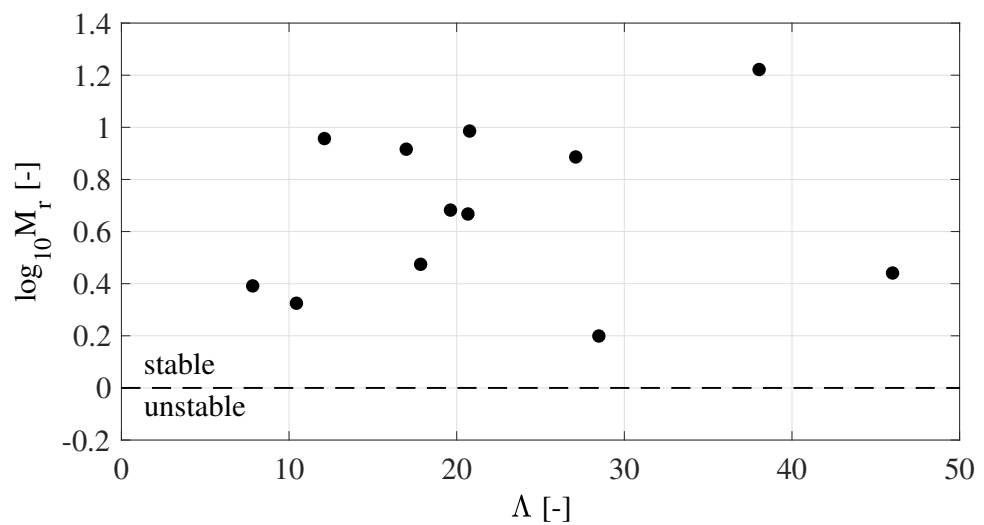

Figure .7: Predicted critical mass ratio against compressibility number for 12 HGJB-supported prototypes designed by the authors 


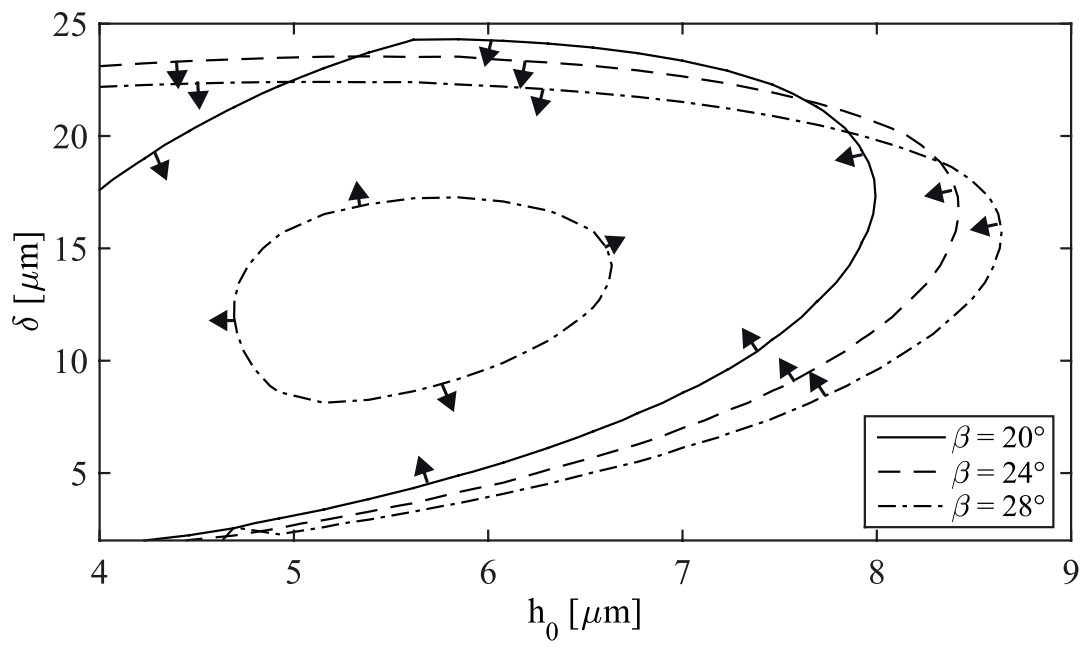

Figure .8: Examples of stability limits for the considered rotor and $\alpha=0.6$. The arrows indicate the orientation of the stable domain 


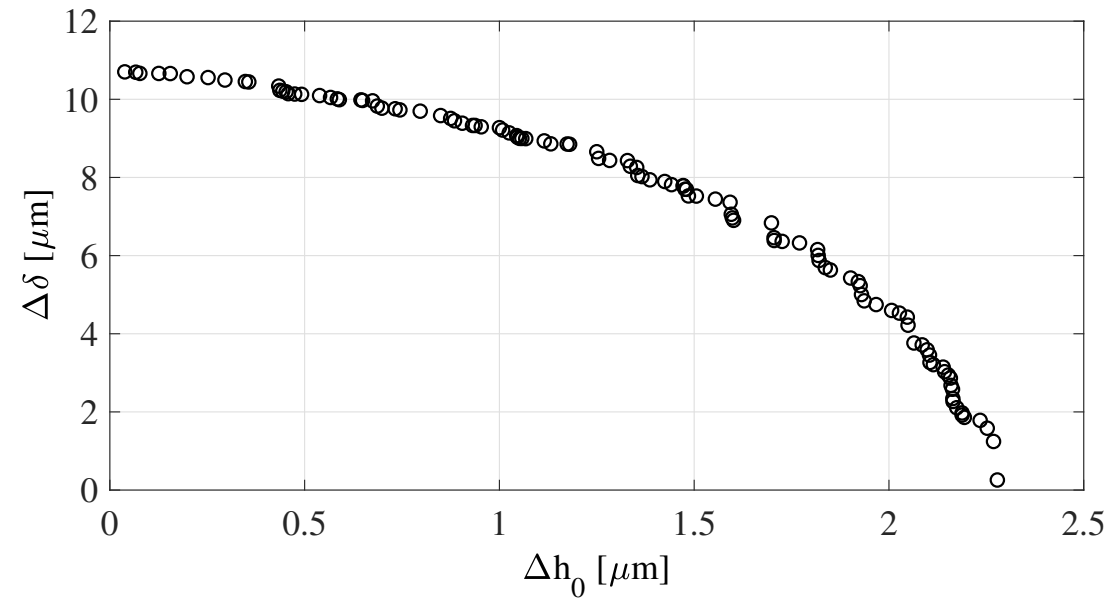

Figure .9: Global Pareto front for the nominal rotor-bearing system 

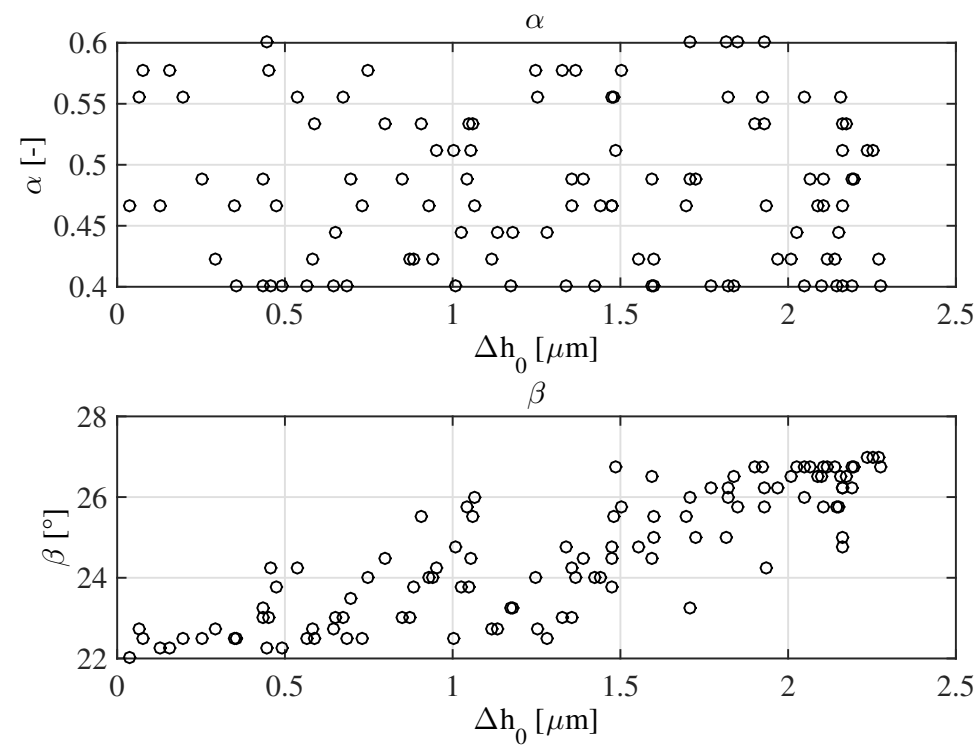

Figure .10: Values of $\alpha$ and $\beta$ resulting in the points shown in the Pareto front of Figure .9. 

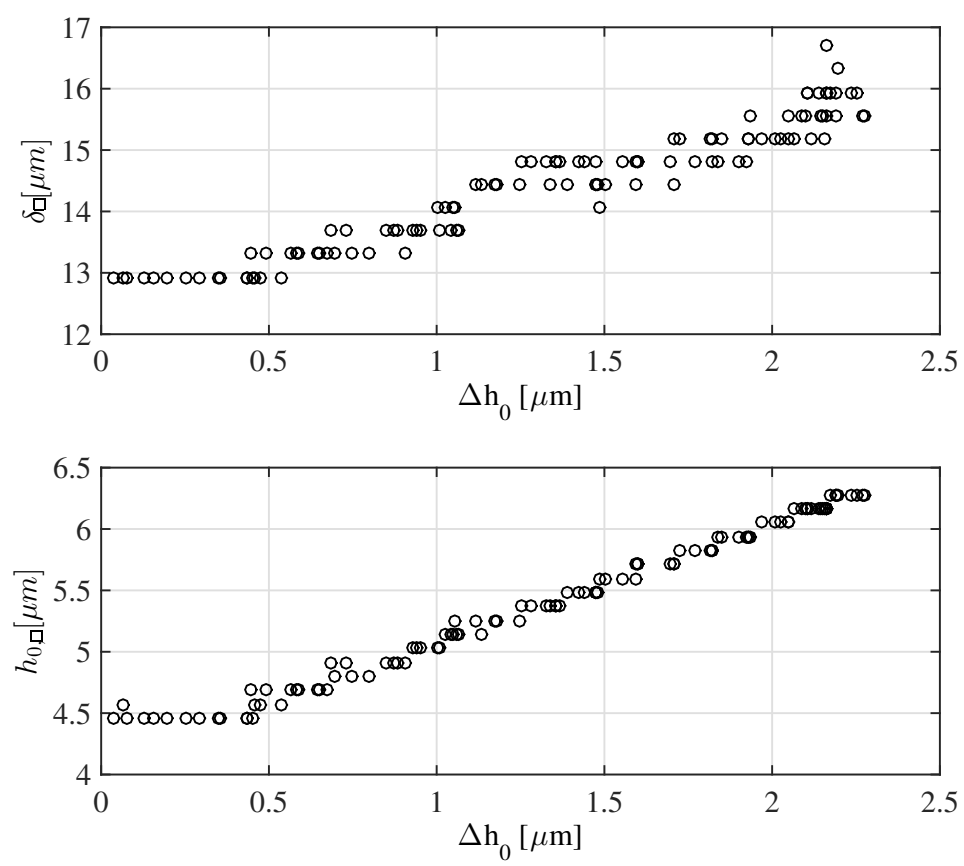

Figure .11: Values of $h_{0, \square}$ and $\delta_{\square}$ resulting in the points shown in the Pareto front of Figure .9. 

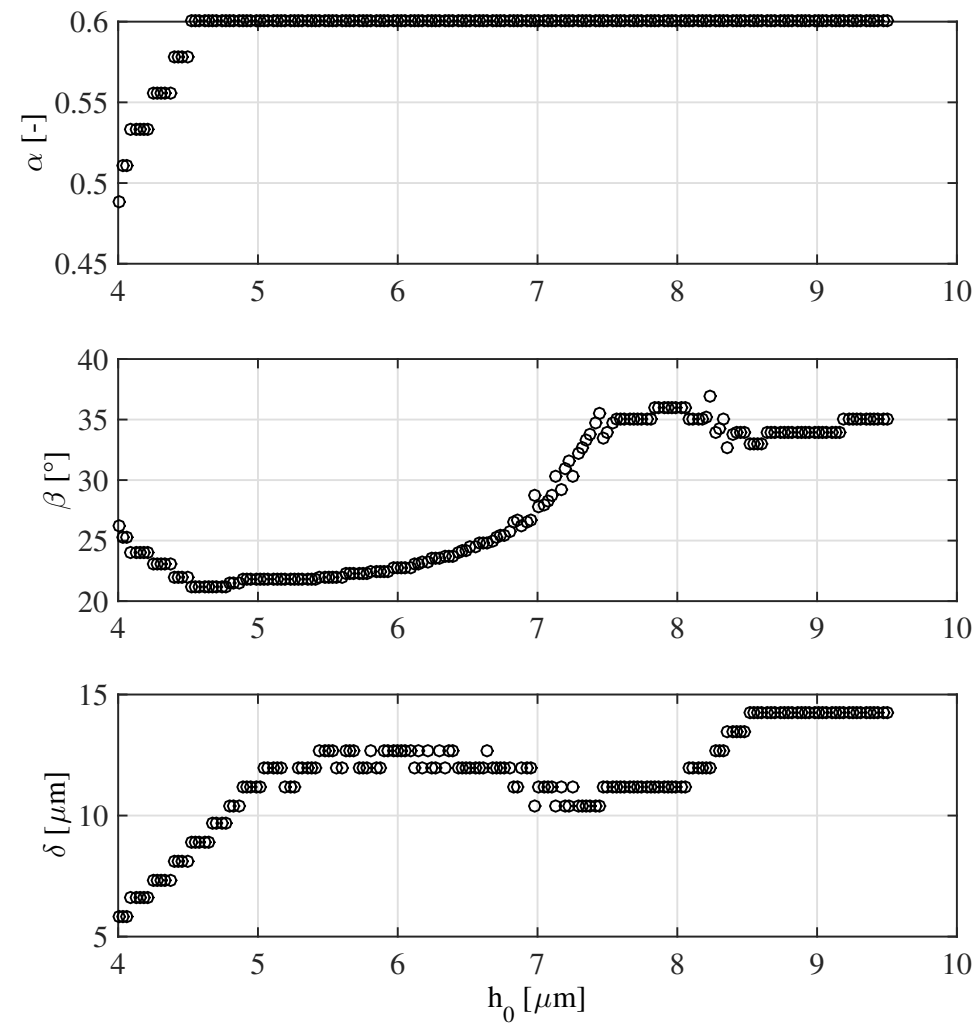

Figure .12: Output parameters from the optimization maximizing the critical mass for different values of $h_{0}$. 


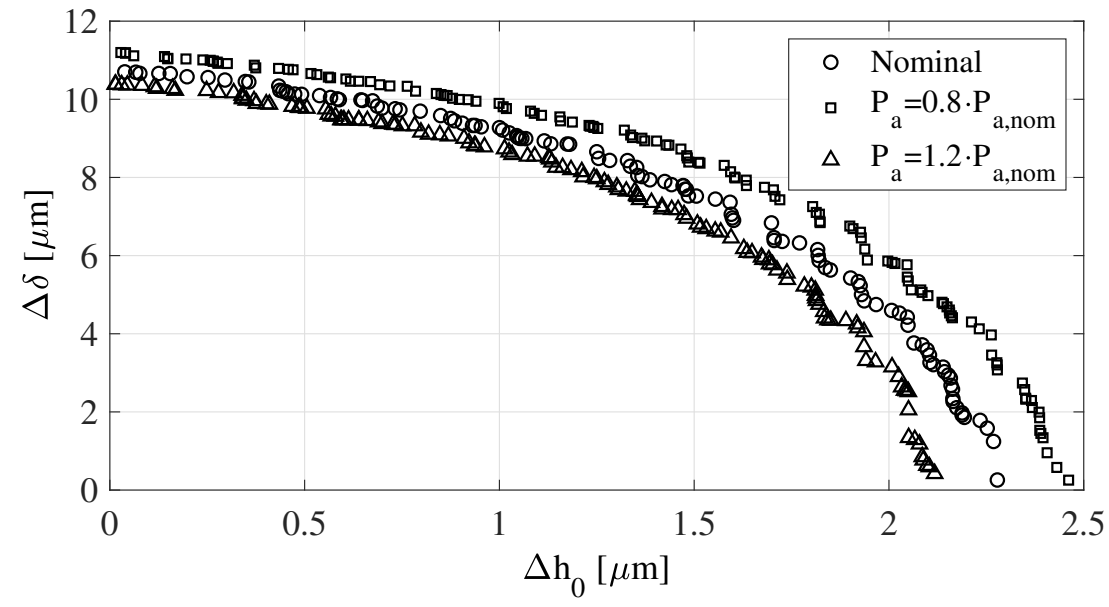

Figure .13: Effect of the ambient pressure on the global Pareto front 


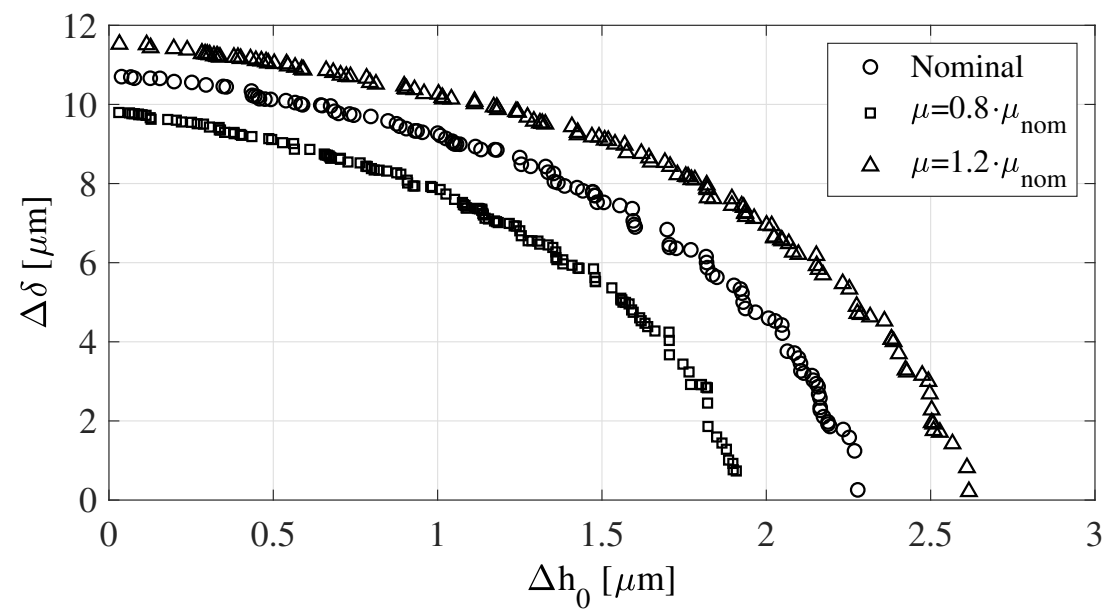

Figure .14: Effect of the gas viscosity on the global Pareto front 


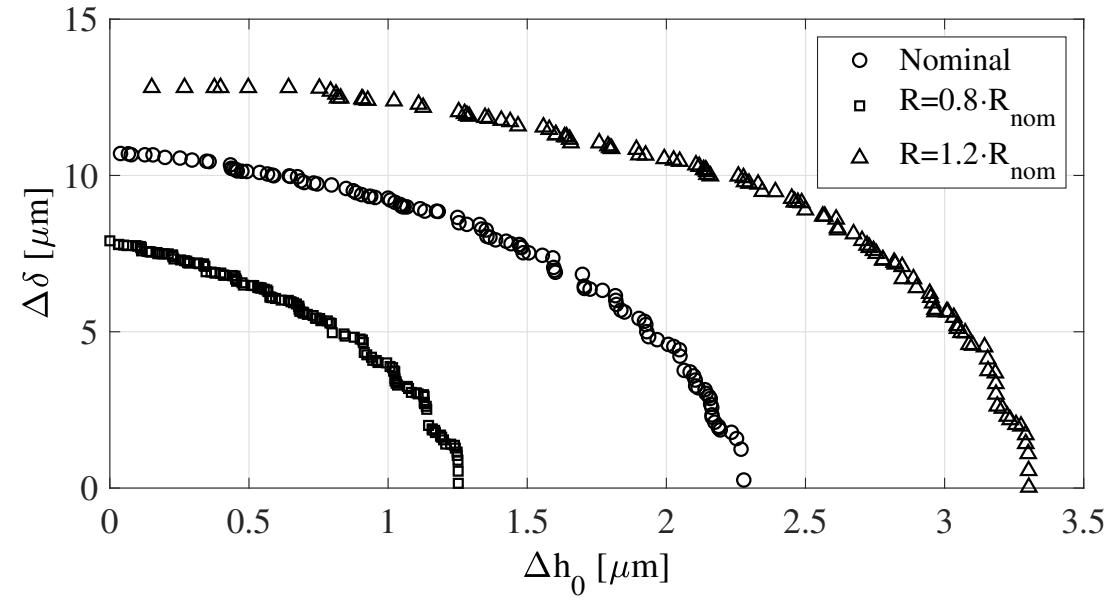

Figure .15: Effect of the rotor radius on the global Pareto front 


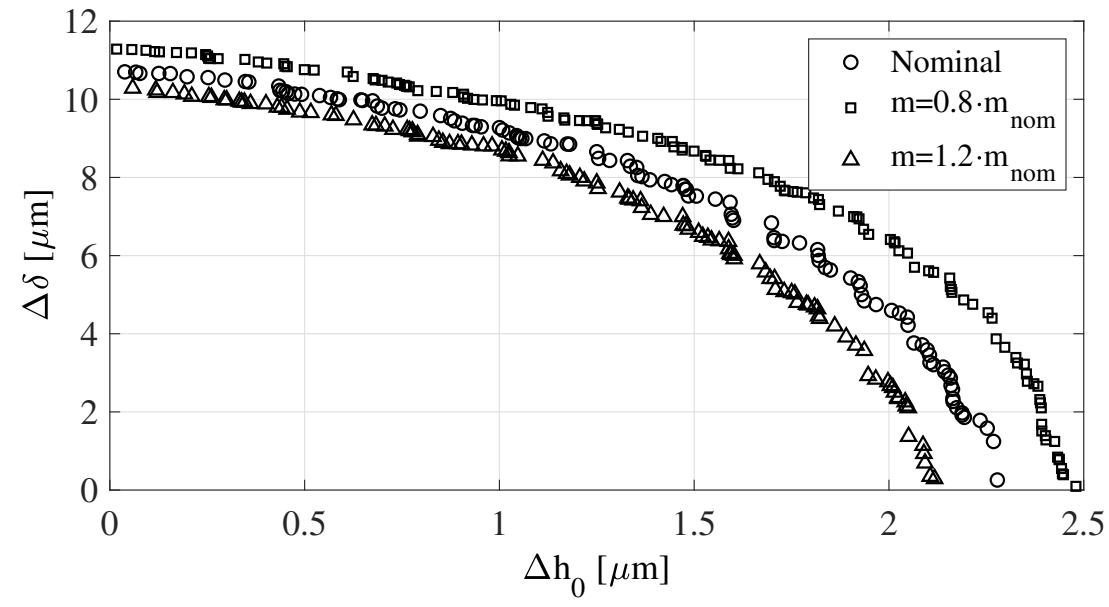

Figure .16: Effect of the rotor mass on the global Pareto front 


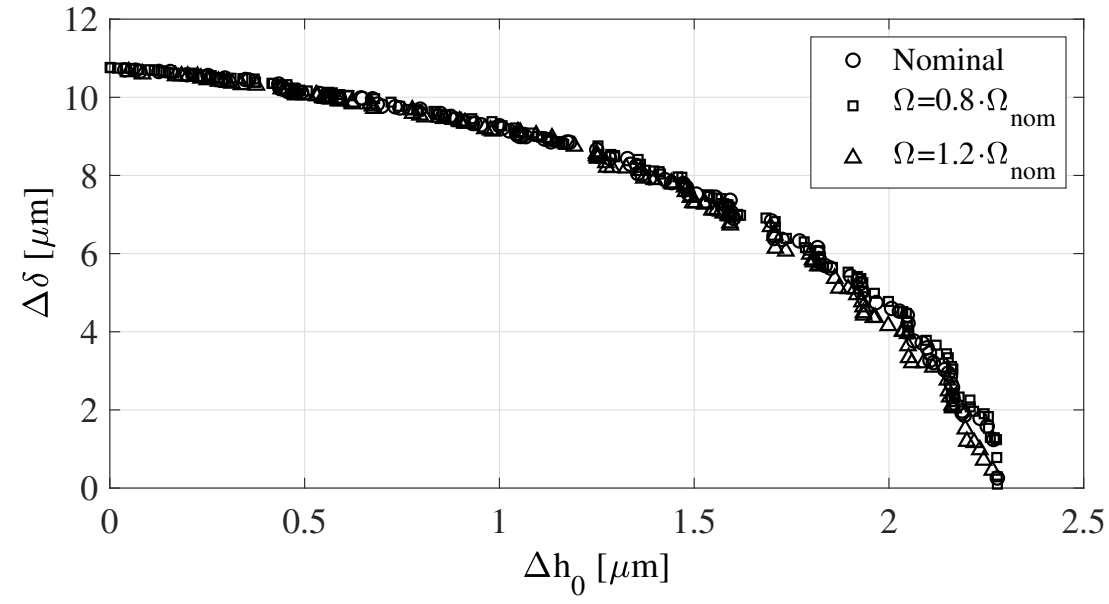

Figure .17: Effect of the rotor maximum speed on the global Pareto front 


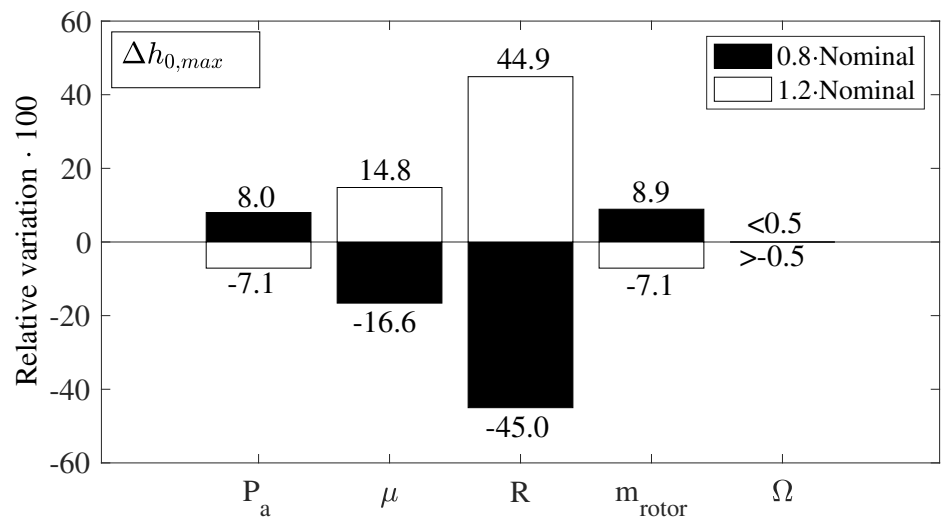

Figure .18: Effect of parametric variation on the maximum clearance tolerance 


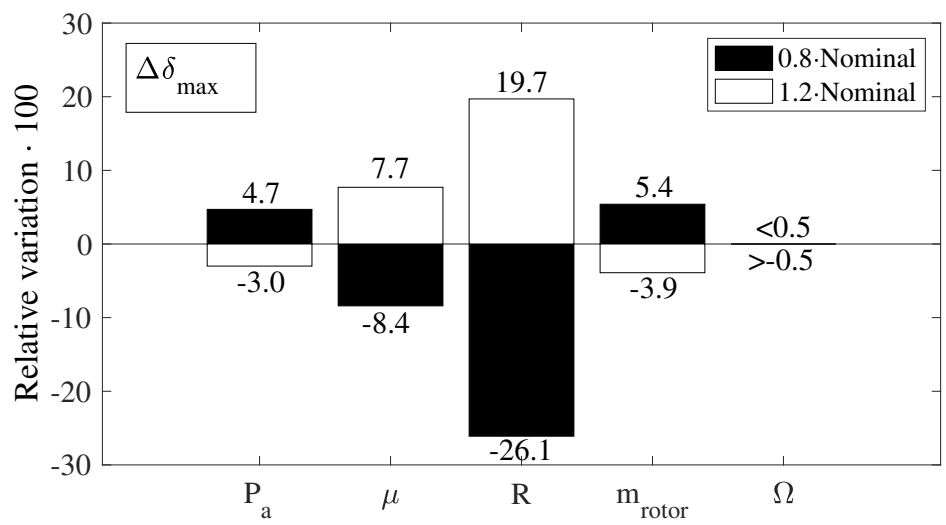

Figure .19: Effect of parametric variation on the maximum groove depth tolerance 


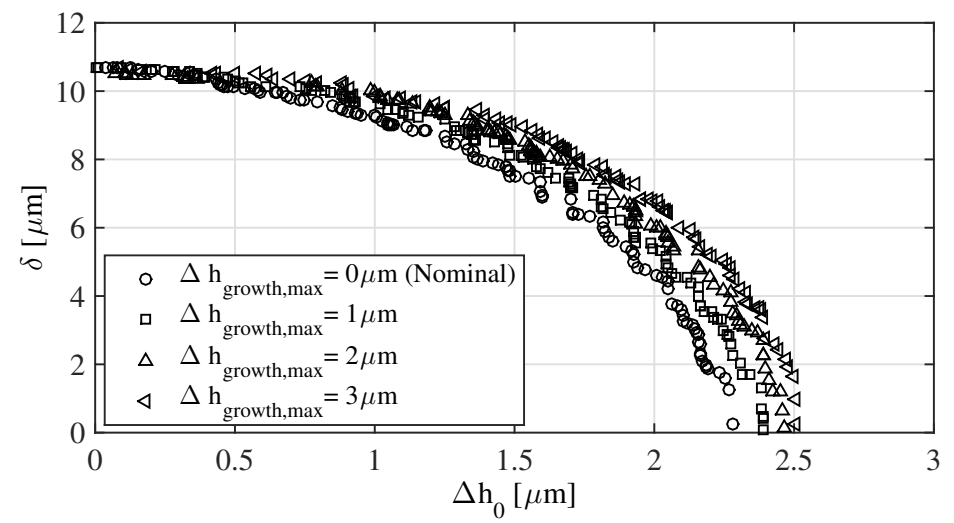

Figure .20: Effect of the centrifugal rotor radial growth on the tolerance Pareto Front 